\title{
Function of Ant Colony Algorithm in Path Optimization of Urban Fire Truck
}

\author{
Shigang WANG ${ }^{1, a}$, Yuchao JIANG ${ }^{1, b}$, Fengjuan WANG ${ }^{1, c}$ \\ ${ }^{1}$ School of Mechatronics Engineering, Qiqihar University, Qiqihar 161006, China \\ aemail: hljwangsg@163.com, bemail: 791062882@qq.com, cemail: wfj320110@163.com
}

Keywords: Ant Colony Algorithm, Path Optimization, Simulation

\begin{abstract}
When the city burst fire, the city fire truck can reach the fire scene in the shortest time to carry out the fire is to reduce the loss of the core. Therefore, this paper introduces ant colony algorithm to solve the core problem, by building the model, using the grid environment information, search for the best parameters of the algorithm, use numerical example simulation to illustrate the ant colony optimization algorithm on fire path has a good value. The results show that the ant colony algorithm can greatly improve the efficiency on this issue, saving time.
\end{abstract}

\section{Introduction}

In all kinds of disasters, fire is one of the most common and most common threats to public safety and social development. When there is a fire, if people not in time to rescue, will give people's lives and property to bring huge losses. The research on the path of the fire truck is in the shortest time, more scientific and effective selection of fire scene to the fire brigade's optimal path, so as to reduce the time used in the road, the fastest to reach the scene of the fire rescue.

Traditional mathematical optimization methods in solving large, complex type of problem will be powerless. In recent years, many scholars use bionic optimization algorithms to solve path problems. In 1995,American scholar Dr. Eberhart and Dr. Kennedy inspired by birds predatory behavior, proposed PSO algorithm can be used to solve the dynamic network of the vehicle path planning [1]. In 2002, Professor Li Xiaolei, associate professor at Shandong University, according to the behavior characteristics of fish populations in the water, studied the artificial fish swarm algorithm, and proposed a new type of top-down optimization model, which can be used to solve the problem of path [2]. In 2005, the Indian scholar Krishnanand and Ghose observed through the analog signal emitted by fireflies at foraging, mating and other behaviors, constructed out of the firefly algorithm, applied to function optimization, robotic path planning [3]. In 2011, Taiwan scholars Pan Wenchao according to the observation of the fruit fly with its own sense and smell, can smell 40 kilometers away from the food and fly to the direction, proposed the fruit fly algorithm, can be used for path optimization [4].

However, because these algorithms are put forward in recent years, many algorithms are still in the simulation stage. There is no perfect theory basis for analysis, and the validity of the algorithm is not given. Therefore, the ant colony algorithm is used to solve the problem of path optimization of urban fire truck. by building the model, using the grid environment information, search for the best parameters of the algorithm, Matlab simulation shows that the ant colony algorithm has a good application value in the path optimization of the fire truck. The results show that the ant colony algorithm can greatly improve the efficiency on this issue, saving time.

\section{Problem Description}

The route choice of the fire truck is described as follows: the fire brigade in receiving the alarm, the fire truck in the shortest time to reach the fire scene. Therefore, the fire center is concerned about the problem can not just stay in the number of vehicles and the number of firefighters how much. In this paper, the fire truck arrived at the scene of the fire at the shortest time for the target, establish the model and suppose to meet the following conditions: 
(1) There is only one fire command center, and the fire engine must be from the command center to the fire scene;

(2) The coordinates of the fire command center and the fire scene are known;

(3) After the completion of the fire fighting mission, the fire truck shall return to the starting place and wait for the new task;

(4) All rescue facilities are complete, and each of the rescue facilities is required to Be rescued by a fire engine;

(5) Do not consider the accident of the fire truck encountered in the rescue.

\section{Ant Colony Algorithm}

Algorithm Description. Colony is also called ant colony optimization, the earliest proposed is by the Italy Dorgio and its research team in early 1990s [5,6]. Since then, the algorithm has been concerned by many scholars and experts, and has been systematically studied by many people. Using ant colony algorithm to solve the traveling salesman problem, distribution, job scheduling problem will get satisfactory results $[7,8]$.

Ant colony algorithm is a probabilistic algorithm, used to find the optimal path in the graph. This is a new general heuristic method for solving combinatorial optimization problems. The algorithm has the advantages of strong advantages, positive feedback and distributed computing, which makes the algorithm can find better solutions faster and easier to implement.

Compared with other algorithms, ant colony algorithm is simple in concept, less parameters, no mutation, crossover, and so on. And the results can be better than some genetic algorithm. The ant colony algorithm has a broad development prospects in both theory and practice. From the proposed to the present, the research of ant colony algorithm in various industries is growing, almost all the optimization field can use the ant colony algorithm, and also appeared the ant colony algorithm simulation software.

Basic Principles of Ant Colony Algorithm. Biologists in the years of painstaking research found, in the nature of ants is no visual sense, the single ant intelligence and ability are small. Simply rely on to communicate with their peers, the special material left behind by the information element, to guide their direction of action, to determine how to walk. A lot of research found that in real life, ants in the food source and the nest almost forming a straight line path, ant group not only can adapt to the environment changes, but also ordered the completed complex task. In the first encounter obstacles, regardless of the path distance, the ants are uniformly distributed action. In the action after the ants will move in the direction of high levels of information. This will lead to new ants in this direction through the left pheromones will increase the original pheromone that is to say that the ants will also be greatly possible to choose the path to walk. Because in the same time the short path will have more ants to walk, then the ants choose a shorter path is more likely, in the end, all the ants will choose the shortest path to walk. A path to select an ant is shown in Figure 1.

From adaptation to cooperation, the ants can get the optimal solution and complete the search of the path.

Ant Colony Algorithm Flow Chart. Recently, the ant colony algorithm is applied in the combination optimization, which is based on the observation of the behavior of ants foraging in nature, therefore, in the construction of the ant colony algorithm search process where we use artificial ants instead of nature ant colony. The basic idea of the ant colony algorithm is this:

(1) Initially, a group of ants go out to find food, then the ants have not passed any path, so at the moment there is no pheromone, ants will just choose to walk the path;

(2) Over time, the next state, each ant at a different point in the initial position to a position between leaving the pheromone, has reached the target of ants start to go back, other ants continue to walk, at this moment, the next batch of ants into action, they will be subject to the ant pheromones affect previously left, according to the high concentration of pheromone walking path;

(3) Then when the next state, just no pheromone ant walked on the path will be volatile for some, but there are ants traversed path pheromone will increase. Later, there will be new ants out of action, repeat (2) step. 
In ant colony algorithm, an ant is not intelligent, in order to distinguish between the nature of the ants, we put the ants in the ant colony algorithm called artificial ants, only the mutual cooperation of the artificial ants can find the solution to the optimization problem. In ant colony algorithm, the change of one state to the next state is called an iteration. After many times of iteration, the pheromone on a path is higher than the other path, which can be used as an optimal path. A basic flow chart ant colony algorithm is shown in Figure 2.

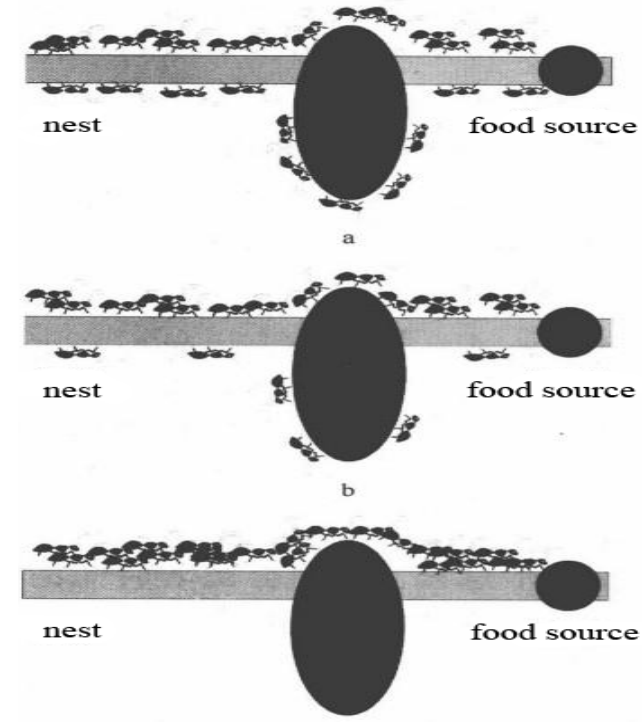

Fig. 1 A path to select an ant

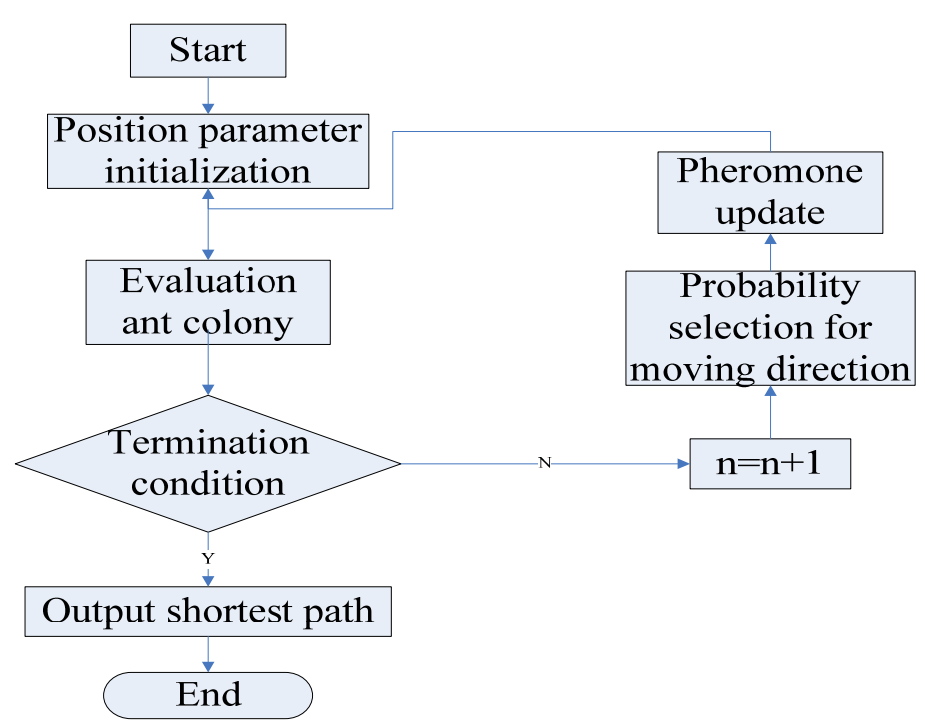

Fig. 2 A basic flow chart ant colony algorithm

Ant Colony Algorithm Modeling and Step. First, set up a fire truck working environment. Set fire engines in the two-dimensional (RS) working environment for rescue. In the working environment of the various corners of the road as a fire truck encountered obstacles. In the course of the fire truck, the obstacle is fixed. The RS is divided by the grid method, fire engines were walking in the divided grid. Raster no obstruction is feasible grid hinder grid is not feasible grid. Fire trucks are in the order of the fire scene from the fire center to the fire, and each grid is marked as $1,2,3, \ldots$, $n$. Let the gate number set is $S, S=\{1,2,3, \ldots, N\}$. According to the corresponding relationship between the above, we can know that the $G(0,0)$ is $1, G(1,0)$ is 2 , until $g(X, Y)$ is $N$.

Matrix consisting of 0 and 1 with a gate of fire format of environmental information, can be represented by 0 grid, not through the grid with 1 to express. Because the grid structure is the most simple and easy to understand in the spatial data structure, and the property is obvious, the position is implicit, based on the location of the row and column number is converted to the corresponding coordinates, so this use Matlab to draw raster. Figure 3 is an obstacle in the path represented by the grid method, in which the black area represents an obstacle.

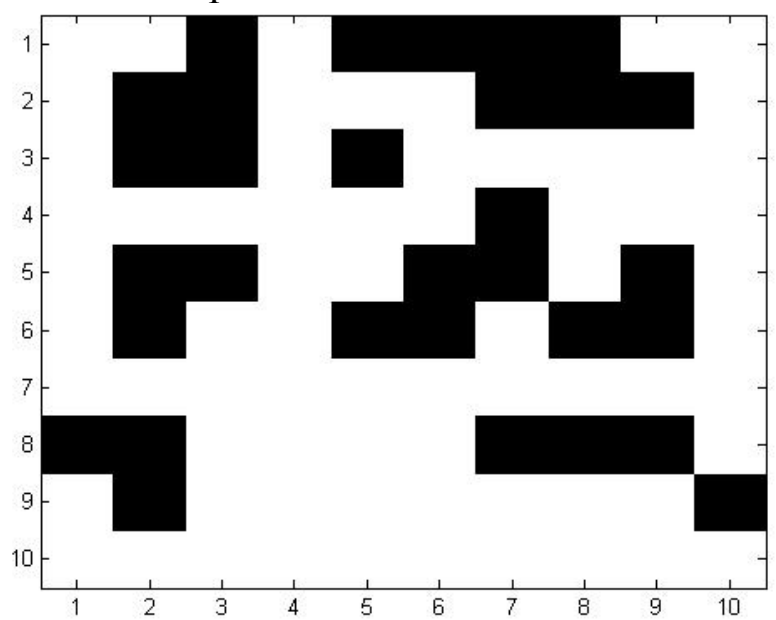

Fig. 3 Path planning grid method 
At the beginning, the path node can be chosen for $D, D=\{0,1, \ldots, N-1\}$. Initially, there are $m$ ants, information inspire factor $\alpha$, the relative importance of the trajectory of representation, reflecting the ants in motion the process of accumulation of pheromones in ants sports role, $\alpha$ is larger, the greater the probability description ants choose this path back; inspired desired factor $\beta$, indicating the relative importance of visibility, reflecting the ants during exercise in inspiration factor $\alpha$ importance in the whole path; heromone evaporation coefficient for $\rho$, is a constant, represents the weight; the number of iterations for $N_{c}$, and have a tabu list. Initialization set $\Phi$, select the starting point $S$ and the destination point $E$ grid number, let ant colony starts walking at the starting point $S$. In good grid points on the grid by the probability ant $i$ to $j$ is $p_{i j}^{k}(t)=\frac{\tau_{i j}^{k}(t) \eta_{i j}^{\beta}(t)}{\sum \tau_{i j}^{\alpha}(t) \eta_{i j}^{\beta}(t)}(\mathrm{j} \in \mathrm{c})$, Otherwise 0 . Among them, $\eta_{i j}(t)=\frac{1}{d_{i j}}$. The formula $d_{i j}$ represents fire truck grid distance from $i$ to $j ; \eta_{i j}$ shows that the fire truck $i$ come from $j$ inspiration level; $\tau_{i j}$ representative pheromone. Every time the ant $k$ is transferred, the node $j$ is added to the tabu list until all the ants in the loop reach the destination, each ant is calculated by taking the path length at the finish line, saved. Update pheromone is $\tau_{i j}(t+1)=\rho \tau_{i j}(t)+\sum_{k=1}^{m} \Delta \tau_{i j}^{k}(t, t+1)$; in formula when an ant $k$ from $i$ through $j$, $\Delta \tau_{i j}^{k}(t)=\frac{Q}{l_{k}}$, Otherwise 0.

\section{Algorithm Application Example}

At present, there are no theoretical basis for the optimal parameters of the ant colony algorithm. So, it is very important to find out the solutions to the $\alpha, \beta, \rho, m$ parameters. So far, most of the parameters are based on the experimental simulation of the statistical data and experience in books. Through several tests, the last in the number of iterations 180 times, set the parameters for $\alpha=1.1, \beta=6, \rho=0.45$. Using Matlab to simulate, find the best way of the fire engine from the fire center to the fire scene. Part of the simulation is shown in Figure 4 and Figure 5.

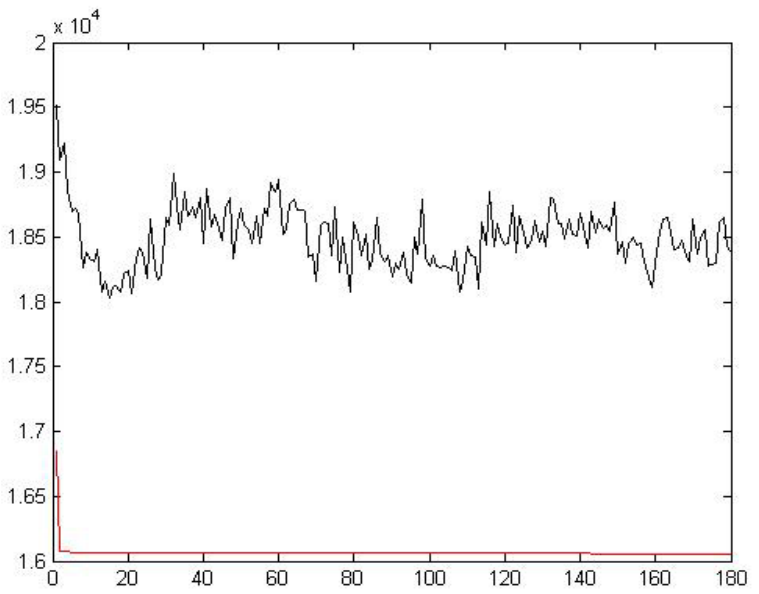

Fig. 4 Each cycle the shortest path length and average path length

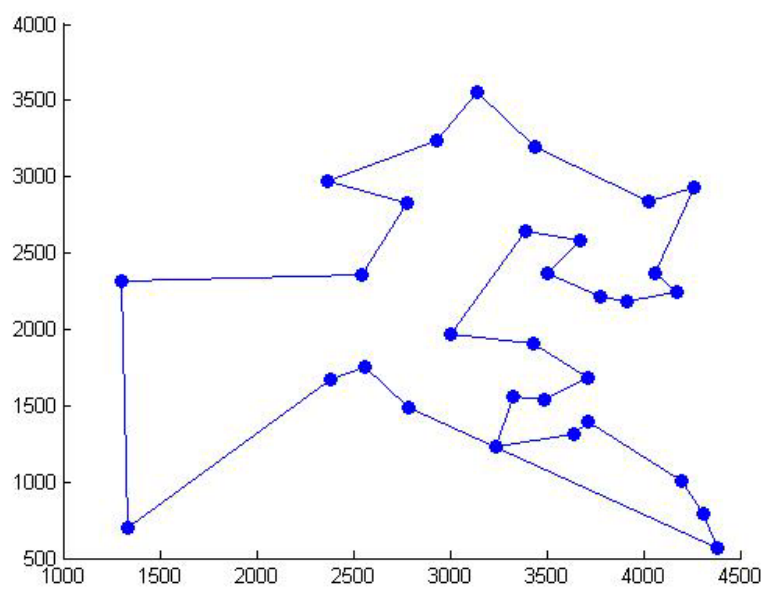

Fig. 5 Iterations shortest path 180 times

\section{Conclusions}

(1) Using the grid method to describe the path of a fire truck straightforward, describing the path in terms of grid method has a very important position.

(2) By setting different iterations, we found that when the number of ants and the number of 
obstacles similar to better use ant colony algorithm to find the optimal solution path in a minimum number of iterations.

(3) When the pheromone accumulated too large, the simulation results are not satisfactory, prone to stagnation.

(4) The pheromone of ant colony has little effect on the ant colony algorithm itself.

(5) Every time a new cycle, the tabu list is empty, so that a new round of ants can random walk, which helps to get more accurate value.

\section{Acknowledgements}

This work was supported by Project of Department of Education of Heilongjiang Province, China (Grant No. 12521597) and Qiqihar University Graduate Innovation Research Project (Grant No. YJSCX2015-ZD15).

\section{References}

[1] Lv Dan, Tong C M, Zhong Weijun. Hybrid particle swarm optimization and, algorithm based on simulated annealing algorithm [J]. Computer Engineering and Design, 201131 (2): 663-666.

[2] Li Xiaolei, F, Qian Jixin. A autonomous animals optimization model based on: fish swarm algorithm [J]. System Engineering Theory and Practice, 200222 (11): 32-38.

[3] Liu Changping, ye Chunming. A novel intelligent bionic algorithm: Firefly algorithm [J], Journal of Computer Research and Application Settings, 201128 (9): 3295-3296.

[4] Han Junying, Liu Chengzhong. Adaptive chaos fruit fly optimization algorithm [J], Journal of Computer Applications, 201333 (5): 1313-1316.

[5] Wang Lei, Wu Qidi. Ant system algorithm in continuous space optimization [J], Control and Decision, 200318 (1): 45-48.

[6] Dorigo M, Maniezzo V, Colorni A. Ant system: optimization by a colony of cooperating agents, Systems, Man, and Cybernetics, Part B: Cybernetics [J]. IEEE Transactions on, 199626 (1): 29-41.

[7] Dorigo M, Gambardella L M. Ant colony system: a cooperative learning approach to the traveling salesman problem [J]. Evolutionary Computation, IEEE Transactions on, 1997 1 (1): 53-66.

[8] Zhou Wei. Dynamical simulation of simple ant systems [J]. Control and Decision, 2003 (3): 317-319. 